Supporting Information for

\title{
Mixed-Ligand Approach to Palladium-Catalyzed Direct Arylation Polymerization: Effective Prevention of Structural Defects Using Diamines
}

Eisuke Iizuka, Masayuki Wakioka, and Fumiyuki Ozawa*

International Research Center for Elements Science (IRCELS), Institute for Chemical Research, Kyoto University, Uji, Kyoto 611-0011, Japan

E-mail: ozawa@scl.kyoto-u.ac.jp

\section{Contents}

$\begin{array}{lr}\text { Experimental Section (Supplementary) } & \text { S2 }\end{array}$

$\begin{array}{ll}\text { General Considerations. } & \text { S2 }\end{array}$

$\begin{array}{ll}\text { Characterization of Poly(DTS-alt-TPD) (3). } & \text { S2 }\end{array}$

$\begin{array}{ll}\text { Preparation of H-TPD- } \mathrm{C}_{6} \mathrm{H}_{4} \mathrm{CF}_{3}(\mathbf{5}) . & \text { S2 }\end{array}$

$\begin{array}{ll}\text { Preparation of Cross-Coupling Product } 6 . & \text { S3 }\end{array}$

$\begin{array}{ll}\text { Preparation of Homocoupling Product } 7 . & \text { S3 }\end{array}$

Table S1. Effects of Coligands (Lc) on the Structures of DTS-alt-TPD Oligomers S4

Formed at an Early Stage of DArP

Table S2. Effects of the Amounts of L4 and L5 on the Structures of DTS-alt-TPD Oligomers S5

Figure S1. ${ }^{1} \mathrm{H}$ NMR spectrum of the DArP product of run 1 in Table $1 . \quad$ S5

Figure S2. ${ }^{1} \mathrm{H}$ NMR spectrum of the DArP product of run 3 in Table 2. S6

Figure S2. ${ }^{1} \mathrm{H}$ NMR spectrum of the DArP product of run 5 in Table 2. S6 


\section{Experimental Section (Supplementary)}

General Considerations. NMR spectra were recorded on a $400 \mathrm{MHz}$ spectrometer $\left({ }^{1} \mathrm{H}\right.$ NMR 400.13 MHz and ${ }^{13} \mathrm{C}$ NMR 100.62 MHz). Chemical shifts are reported in $\delta(\mathrm{ppm})$, referenced to ${ }^{1} \mathrm{H}$ (residual) and ${ }^{13} \mathrm{C}$ signals of deuterated solvents as internal standards. Analytical GPC was performed with TOSOH TSKgel GMHHR-H(S)HT columns and $o$-dichlorobenzene as a mobile phase $(1.0 \mathrm{~mL}$ $\left.\min ^{-1}\right)$ at $140{ }^{\circ} \mathrm{C}$. The columns were calibrated against 10 standard polystyrene samples $\left(M_{\mathrm{n}}=1200\right.$ 1410000). Recycling preparative GPC was carried out using two preparative GPC columns (JAIGEL $1 \mathrm{H}-40$ and $2 \mathrm{H}-40)$ and chloroform as the mobile phase $\left(3.8 \mathrm{~mL} \mathrm{~min}^{-1}\right)$ at room temperature. UV-vis absorption spectra were measured in $o$-dichlorobenzene at room temperature.

Characterization of Poly(DTS-alt-TPD) (3). Oligomeric and polymeric products of 3 were characterized by GPC $\left(o-\mathrm{Cl}_{2} \mathrm{C}_{6} \mathrm{H}_{4}, 140{ }^{\circ} \mathrm{C}\right),{ }^{1} \mathrm{H}$ NMR $\left(\mathrm{C}_{2} \mathrm{D}_{2} \mathrm{Cl}_{4}, 130{ }^{\circ} \mathrm{C}\right)$, and MALDI-TOF-MS analysis, as previously reported: Iizuka, E.; Wakioka, M.; Ozawa, F. Macromolecules 2015, 48, 2989. The ${ }^{1} \mathrm{H}$ NMR spectra of the products of runs 3 and 5 in Table 2 are given in Figures S2 and S3, respectively. The NMR data are as follows $\left(\mathrm{C}_{2} \mathrm{D}_{2} \mathrm{Cl}_{4}, 130^{\circ} \mathrm{C}\right)$ : DTS-alt-TPD units: $\delta 8.14-8.08(\mathrm{~m}, 2 \mathrm{H}$, A), 3.78-3.61 (m, 2H, NCH2), 1.83-0.75 (m, 49H, alkyl); terminal groups: $\delta$ 7.98-7.93 (m, a), 7.58 (s,

b), $7.32\left(\mathrm{~d},{ }^{3} J_{\mathrm{HH}}=4.8 \mathrm{~Hz}, \mathbf{c}\right), 7.26(\mathrm{~s}, \mathbf{d}), 7.12\left(\mathrm{~d},{ }^{3} J_{\mathrm{HH}}=4.8 \mathrm{~Hz}, \mathbf{e}\right)$; homocoupling and branching defects: $\delta 8.08-7.98(\mathrm{~m}, \mathbf{B}), 7.47-7.28$ (br, D), 7.23-7.19 (m, C).

Preparation of H-TPD- $\mathbf{C}_{6} \mathbf{H}_{4} \mathbf{C F}_{3}$ (5). This compound was prepared by direct arylation of TPD-H (2) with 1-bromo-4-trifluoromethylbenzene (S1).

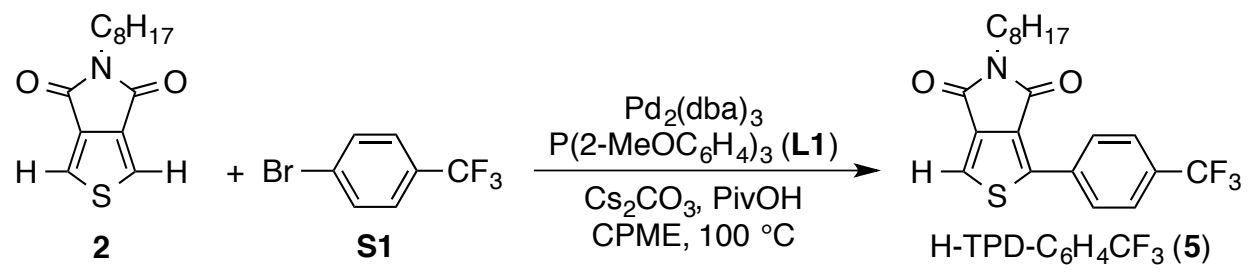

A $50 \mathrm{~mL}$ Schlenk tube was charged with 2 (4.71 g, $17.8 \mathrm{mmol}), \mathbf{S 1}(1.13 \mathrm{~g}, 5.0 \mathrm{mmol}), \mathrm{Cs}_{2} \mathrm{CO}_{3}$ (4.89 g, $15 \mathrm{mmol}$ ), pivalic acid (510 mg, $5.0 \mathrm{mmol}), \mathrm{Pd}_{2}(\mathrm{dba})_{3} \cdot \mathrm{CHCl}_{3}(26.0 \mathrm{mg}, 0.025 \mathrm{mmol}), \mathbf{L 1}$ (35.2 $\mathrm{mg}, 0.10 \mathrm{mmol}$ ), and cyclopentyl methyl ether (CPME, $20 \mathrm{~mL}$ ). The mixture was stirred at room temperature for $30 \mathrm{~min}$, and then at $100{ }^{\circ} \mathrm{C}$ for $22 \mathrm{~h}$. After the mixture was cooled to room temperature, water $(100 \mathrm{~mL})$ was added, and the resulting mixture was extracted with $\mathrm{CHCl}_{3}(3 \times 50 \mathrm{~mL})$. The combined extracts were washed with brine $(100 \mathrm{~mL})$, dried over $\mathrm{MgSO}_{4}$, and concentrated under reduced pressure. The residue was purified by column chromatography $\left(\mathrm{SiO}_{2}\right.$, hexane $/ \mathrm{CH}_{2} \mathrm{Cl}_{2}=4 / 1$ and then 2/1). The crude product was purified by recrystallization from $\mathrm{CHCl}_{3} / \mathrm{MeOH}$ at $-20{ }^{\circ} \mathrm{C}$ to afford $5\left(\mathrm{H}-\mathrm{TPD}-\mathrm{C}_{6} \mathrm{H}_{4} \mathrm{CF}_{3}\right)$ as yellow needles $(1.31 \mathrm{~g}, 64 \%) .{ }^{1} \mathrm{H} \mathrm{NMR}\left(\mathrm{CDCl}_{3}\right): \delta 8.23\left(\mathrm{~d}, J_{\mathrm{HH}}=8.6 \mathrm{~Hz}\right.$, $2 \mathrm{H}), 7.79(\mathrm{~s}, 1 \mathrm{H}), 7.72\left(\mathrm{~d}, J_{\mathrm{HH}}=8.6 \mathrm{~Hz}, 2 \mathrm{H}\right), 3.65\left(\mathrm{t}, J_{\mathrm{HH}}=7.4 \mathrm{~Hz}, 2 \mathrm{H}\right), 1.72-1.62(\mathrm{~m}, 2 \mathrm{H}), 1.40-1.20$ 
$(\mathrm{m}, 10 \mathrm{H}), 0.87\left(\mathrm{t}, J_{\mathrm{HH}}=7.0 \mathrm{~Hz}, 3 \mathrm{H}\right) .{ }^{13} \mathrm{C}\left\{{ }^{1} \mathrm{H}\right\} \mathrm{NMR}\left(\mathrm{CDCl}_{3}\right): \delta 163.2,162.5,145.5,138.5,134.1$, $131.9\left(\mathrm{q},{ }^{2} J_{\mathrm{CF}}=33 \mathrm{~Hz}\right), 130.8,128.4,126.3\left(\mathrm{q},{ }^{3} J_{\mathrm{CF}}=4 \mathrm{~Hz}\right), 124.4,124.0\left(\mathrm{q},{ }^{1} J_{\mathrm{CF}}=272 \mathrm{~Hz}\right), 38.9,32.0$, 29.4, 29.4, 28.7, 27.1, 22.8, 14.3. Anal. Calcd for $\mathrm{C}_{21} \mathrm{H}_{22} \mathrm{~F}_{3} \mathrm{NO}_{2} \mathrm{~S}: \mathrm{C}, 61.60 ; \mathrm{H}, 5.42 ; \mathrm{N}, 3.42$. Found: $\mathrm{C}$, $61.53 ; \mathrm{H}, 5.48 ; \mathrm{N}, 3.36$.

Preparation of Cross-Coupling Product 6. This compound was prepared by direct arylation of HTPD- $\mathrm{C}_{6} \mathrm{H}_{4} \mathrm{CF}_{3}(\mathbf{5})$ with PhI.

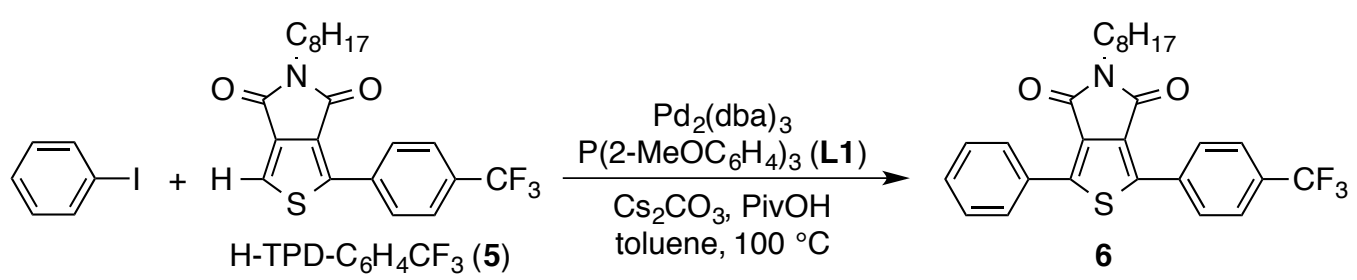

A mixture of 5 (205 mg, $0.50 \mathrm{mmol})$, PhI (102 mg, $0.50 \mathrm{mmol}), \mathrm{Pd}_{2}(\mathrm{dba})_{3} \cdot \mathrm{CHCl}_{3}(2.60 \mathrm{mg}, 2.5$ $\mu \mathrm{mol}), \mathbf{L 1}(3.52 \mathrm{mg}, 10 \mu \mathrm{mol}), \mathrm{Cs}_{2} \mathrm{CO}_{3}(489 \mathrm{mg}, 1.5 \mathrm{mmol})$, and pivalic acid $(51.1 \mathrm{mg}, 0.50 \mathrm{mmol}) \mathrm{in}$ toluene $(1.0 \mathrm{~mL})$ was stirred at room temperature for $30 \mathrm{~min}$, and then at $100{ }^{\circ} \mathrm{C}$ for $13 \mathrm{~h}$. The mixture was cooled to room temperature, diluted with $\mathrm{CHCl}_{3}(30 \mathrm{~mL})$, and washed with water $(3 \times 5 \mathrm{~mL})$ and $5 N \mathrm{HCl}(5 \mathrm{~mL})$. The organic phase was concentrated under reduced pressure. The residue was purified by column chromatography $\left(\mathrm{SiO}_{2}\right.$, hexane $/ \mathrm{CH}_{2} \mathrm{Cl}_{2}=4 / 1,3 / 1$, and then 2/1) to afford cross-coupling product 6 as a white solid (233 $\mathrm{mg}, 0.46 \mathrm{mmol}, 92 \%)$, along with a small amount of homocoupling product 7 as a yellow solid (1.1 mg, $4.2 \mu \mathrm{mol}, 1 \%) .6$ : ${ }^{1} \mathrm{H} \mathrm{NMR}\left(\mathrm{CDCl}_{3}\right): \delta 8.21\left(\mathrm{~d}, J_{\mathrm{HH}}=8.3 \mathrm{~Hz}, 2 \mathrm{H}\right)$, $8.09\left(\mathrm{~d}, J_{\mathrm{HH}}=8.3 \mathrm{~Hz}, 2 \mathrm{H}\right), 7.67\left(\mathrm{~d}, J_{\mathrm{HH}}=8.3 \mathrm{~Hz}, 2 \mathrm{H}\right), 7.47-7.40(\mathrm{~m}, 3 \mathrm{H}), 3.61\left(\mathrm{t}, J_{\mathrm{HH}}=7.4 \mathrm{~Hz}, 2 \mathrm{H}\right)$, $1.70-1.63(\mathrm{~m}, 2 \mathrm{H}), 1.33-1.24(\mathrm{~m}, 10 \mathrm{H}), 0.89\left(\mathrm{t}, J_{\mathrm{HH}}=6.7 \mathrm{~Hz}, 3 \mathrm{H}\right) .{ }^{13} \mathrm{C}\left\{{ }^{1} \mathrm{H}\right\} \mathrm{NMR}\left(\mathrm{CDCl}_{3}\right): \delta 162.6$, 162.6, 146.0, 142.2, 133.8, 132.0, 131.5 (q, ${ }^{2} J_{\mathrm{CF}}=32 \mathrm{~Hz}$ ), 130.6, 130.5, 130.2, 129.1, 128.4, 128.3, 128.1, $125.9\left(\mathrm{q},{ }^{3} J_{\mathrm{CF}}=4 \mathrm{~Hz}\right), 123.9\left(\mathrm{q},{ }^{1} J_{\mathrm{CF}}=272 \mathrm{~Hz}\right), 38.8,31.9,29.3,29.3,28.5,27.1,22.7,14.1$. Anal. Calcd for $\mathrm{C}_{27} \mathrm{H}_{26} \mathrm{~F}_{3} \mathrm{NO}_{2} \mathrm{~S}$ : C, 66.79; H, 5.40; N, 2.88. Found: C, 66.85; H, 5.41; N, 2.94 .

Preparation of Homocoupling Product 7. This compound was prepared by oxidative dimerization of $\mathrm{H}-\mathrm{TPD}-\mathrm{C}_{6} \mathrm{H}_{4} \mathrm{CF}_{3}(\mathbf{5})$.

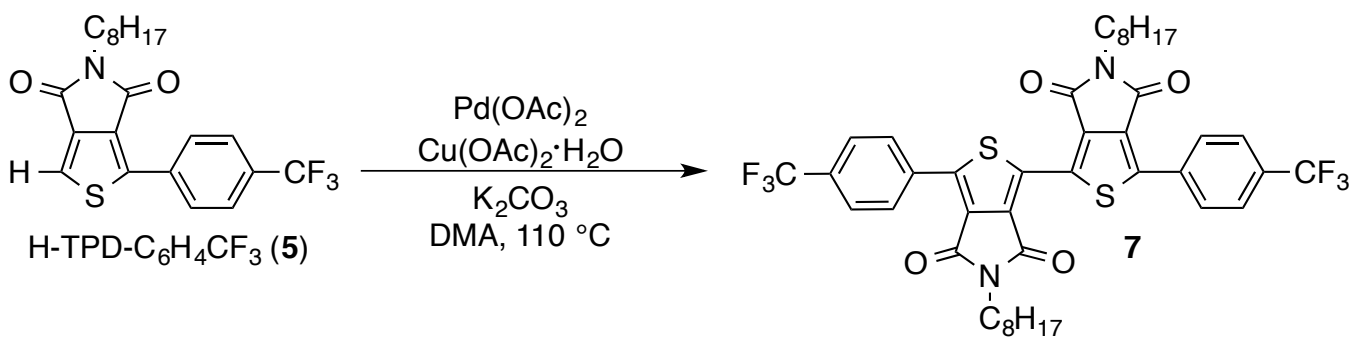

A mixture of 5 (H-TPD- $\left.\mathrm{C}_{6} \mathrm{H}_{4} \mathrm{CF}_{3} ; 16.4 \mathrm{mg}, 40 \mu \mathrm{mol}\right), \mathrm{Cu}(\mathrm{OAc})_{2} \cdot \mathrm{H}_{2} \mathrm{O}(16.8 \mathrm{mg}, 84 \mu \mathrm{mol})$, and $\mathrm{K}_{2} \mathrm{CO}_{3}(11.9 \mathrm{mg}, 86 \mu \mathrm{mol})$ in DMA $(0.64 \mathrm{~mL})$ was heated at $110^{\circ} \mathrm{C}$ for $10 \mathrm{~min}$ in a gas-tight Schlenk tube equipped with a Teflon stopcock. A solution of $\mathrm{Pd}(\mathrm{OAc})_{2}(6.15 \mathrm{mg}, 27 \mu \mathrm{mol})$ in DMA $(0.36 \mathrm{~mL})$ 
was added, and the reaction mixture was stirred at $110^{\circ} \mathrm{C}$ for $5 \mathrm{~h}$, and then cooled to room temperature. The reaction solution was diluted with $\mathrm{CHCl}_{3}(5 \mathrm{~mL})$, and washed with water $(3 \times 5 \mathrm{~mL})$. The organic phase was concentrated to dryness under reduced pressure. The residue was purified by column chromatography $\left(\mathrm{SiO}_{2}\right.$, hexane $\left./ \mathrm{CH}_{2} \mathrm{Cl}_{2}=1 / 1\right)$ to afford homocoupling product 7 as a yellow solid (7.10 mg, $8.7 \mu \mathrm{mol}, 44 \%) .{ }^{1} \mathrm{H} \mathrm{NMR}\left(\mathrm{CDCl}_{3}\right): \delta 8.31\left(\mathrm{~d}, J_{\mathrm{HH}}=8.3 \mathrm{~Hz}, 4 \mathrm{H}\right), 7.75\left(\mathrm{~d}, J_{\mathrm{HH}}=8.3 \mathrm{~Hz}, 4 \mathrm{H}\right), 3.70$ $\left(\mathrm{t}, J_{\mathrm{HH}}=7.2 \mathrm{~Hz}, 4 \mathrm{H}\right), 1.73-1.68(\mathrm{~m}, 4 \mathrm{H}), 1.40-1.21(\mathrm{~m}, 20 \mathrm{H}), 0.87\left(\mathrm{t}, J_{\mathrm{HH}}=6.6 \mathrm{~Hz}, 6 \mathrm{H}\right) .{ }^{13} \mathrm{C}\left\{{ }^{1} \mathrm{H}\right\}$ NMR $\left(\mathrm{CDCl}_{3}\right): \delta 162.4,162.3,145.9,134.5,133.3,132.4\left(\mathrm{q},{ }^{2} J_{\mathrm{CF}}=33 \mathrm{~Hz}\right), 131.7,130.9,128.9,126.2$ $\left(\mathrm{q},{ }^{3} J_{\mathrm{CF}}=4 \mathrm{~Hz}\right), 123.8\left(\mathrm{q},{ }^{1} J_{\mathrm{CF}}=272 \mathrm{~Hz}\right), 39.1,31.9,29.3,29.3,28.6,27.1,22.8,14.2$. Anal. Calcd for $\mathrm{C}_{42} \mathrm{H}_{42} \mathrm{~F}_{6} \mathrm{~N}_{2} \mathrm{O}_{4} \mathrm{~S}_{2}: \mathrm{C}, 61.75 ; \mathrm{H}, 5.18 ; \mathrm{N}, 3.43$. Found: C, 61.27; H, 5.45; N, 3.34.

Table S1. Effects of Coligands (Lc) on the Structures of DTS-alt-TPD Oligomers Formed at an Early Stage of DArP (Supplementary) ${ }^{a}$

\begin{tabular}{|c|c|c|c|c|c|c|c|}
\hline run & Lc & $\begin{array}{l}\text { time } \\
(\mathrm{min})\end{array}$ & $\begin{array}{l}\text { yield } \\
(\%)^{b}\end{array}$ & $M_{\mathrm{n}}^{c}$ & $M_{\mathrm{w}} / M_{\mathrm{n}}^{c}$ & DTS-TPD/TPD-TPD ${ }^{d}$ & $\begin{array}{l}\text { DTS-I/DTS-H } \\
\text { /TPD-H }\end{array}$ \\
\hline 1 & L8 & 20 & 81 & 3,700 & 1.71 & $12.8 / \mathbf{0 . 1 9}(1.5 \%)$ & $0.84 / \mathbf{0 . 1 4} / 1.01$ \\
\hline $2^{f}$ & L9 & 1440 & 0 & - & - & - & - \\
\hline 3 & L10 & 1440 & 44 & 2,500 & 1.50 & $5.67 / 0.11(1.9 \%)$ & $1.02 / \mathbf{0 . 1 5} / 0.83$ \\
\hline $4^{f}$ & L11 & 1440 & 0 & - & - & - & - \\
\hline 5 & L12 & 20 & 62 & 2,800 & 1.55 & $8.98 / 0.24(2.7 \%)$ & $0.74 / \mathbf{0 . 1 9} / 1.07$ \\
\hline 6 & L13 & 360 & 79 & 4,200 & 1.69 & $11.5 / \mathbf{0 . 2 6}(2.2 \%)$ & $0.90 / \mathbf{0 . 1 4} / 0.96$ \\
\hline $7^{f}$ & L14 & 1440 & 0 & - & - & - & - \\
\hline 8 & L15 & 150 & 42 & 2,000 & 1.40 & $7.73 / 0.09(1.1 \%)$ & $0.95 / \mathbf{0 . 0 8} / 0.97$ \\
\hline
\end{tabular}

${ }^{a}$ Reactions were run in toluene $(0.4 \mathrm{~mL})$ at $100{ }^{\circ} \mathrm{C}$ using $1(0.2 \mathrm{mmol}), 2$ (0.2 mmol), PivOH (0.2 $\mathrm{mmol}), \mathrm{Cs}_{2} \mathrm{CO}_{3}(0.6 \mathrm{mmol}), \mathrm{Pd}_{2}(\mathrm{dba})_{3}(1.0 \mu \mathrm{mol}, 0.5 \mathrm{~mol} \%), \mathrm{L1}(2 \mathrm{~mol} \%)$, and $\mathbf{L c}(10 \mathrm{~mol} \%)$. ${ }^{b}$ Isolated yield after reprecipitation with $\mathrm{MeOH}$. ${ }^{c}$ Estimated by GPC calibration based on polystyrene standards $\left(o-\mathrm{Cl}_{2} \mathrm{C}_{6} \mathrm{H}_{4}, 140{ }^{\circ} \mathrm{C}\right) .{ }^{d}$ The average number of cross-coupling (DTS-TPD) and homocoupling (TPD-TPD) bonds in each molecule. The ratios of homocoupling bonds are in parentheses. ${ }^{e}$ The average number of end groups in each molecule. ${ }^{f}$ Polymerization did not proceed.

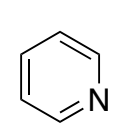

L8

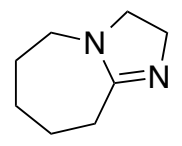

L9

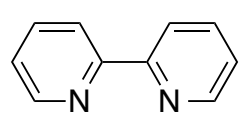

L13

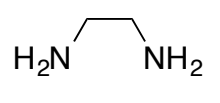

L10

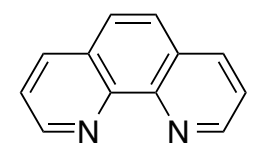

L14

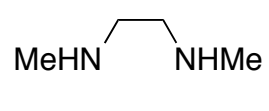

L11

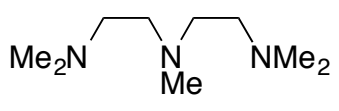

L15 
Table S2. Effects of the Amount of L4/L5 on the Structures of DTS-alt-TPD Oligomers Formed at an Early Stage of DArP ${ }^{a}$

\begin{tabular}{|c|c|c|c|c|c|c|c|}
\hline run & $\begin{array}{l}\text { Lc } \\
(\mathrm{mol} \%)\end{array}$ & $\begin{array}{l}\text { time } \\
(\mathrm{min})\end{array}$ & $\begin{array}{l}\text { yield } \\
(\%)^{b}\end{array}$ & $M_{\mathrm{n}}^{c}$ & $M_{\mathrm{w}} / M_{\mathrm{n}}{ }^{c}$ & DTS-TPD/TPD-TPD ${ }^{d}$ & $\begin{array}{l}\text { DTS-I/DTS-H } \\
\text { /TPD-H }\end{array}$ \\
\hline 1 & L4 (10) & 20 & 40 & 1,900 & 1.41 & $6.87 / 0.08(1.2 \%)$ & $0.90 / \mathbf{0 . 0 9} / 1.01$ \\
\hline 2 & L4 (30) & 35 & 52 & 2,100 & 1.42 & $7.18 / 0.09(1.2 \%)$ & $0.83 / \mathbf{0 . 1 0} / 1.07$ \\
\hline 3 & L4 (50) & 35 & 48 & 2,100 & 1.44 & $7.17 / 0.09(1.2 \%)$ & $0.84 / \mathbf{0 . 1 2} / 1.04$ \\
\hline 4 & $\mathbf{L 5}(5)$ & 60 & 66 & 3,000 & - & $9.11 / \mathbf{0 . 2 0}(2.2 \%)$ & $0.81 / \mathbf{0 . 1 3} / 1.06$ \\
\hline 5 & L5 (10) & 120 & 54 & 2,500 & 1.55 & $7.05 / \mathbf{0 . 0 8}(1.1 \%)$ & $0.86 / \mathbf{0 . 1 1} / 1.03$ \\
\hline 6 & L5 (30) & 180 & 37 & 2,000 & 1.69 & $5.13 / 0.07(1.2 \%)$ & $0.90 / \mathbf{0 . 0 7} / 1.03$ \\
\hline 7 & $\mathbf{L 5}(50)$ & 1440 & 45 & 2,700 & 1.40 & $6.75 / 0.12(1.8 \%)$ & $0.95 / \mathbf{0 . 1 5} / 0.90$ \\
\hline
\end{tabular}

${ }^{a}$ Reactions were run in toluene $(0.4 \mathrm{~mL})$ at $100{ }^{\circ} \mathrm{C}$ using $1(0.2 \mathrm{mmol}), 2$ (0.2 mmol), PivOH (0.2 mmol), $\mathrm{Cs}_{2} \mathrm{CO}_{3}(0.6 \mathrm{mmol}), \mathrm{Pd}_{2}(\mathrm{dba})_{3}(1.0 \mu \mathrm{mol}, 0.5 \mathrm{~mol} \%), \mathbf{L 1}(2 \mathrm{~mol} \%)$, and $\mathbf{L 4}$ or $\mathbf{L 5} .{ }^{b}$ Isolated yield after reprecipitation with $\mathrm{MeOH}$. ${ }^{c}$ Estimated by GPC calibration based on polystyrene standards $\left(o-\mathrm{Cl}_{2} \mathrm{C}_{6} \mathrm{H}_{4}, 140{ }^{\circ} \mathrm{C}\right) .{ }^{d}$ The average number of cross-coupling (DTS-TPD) and homocoupling (TPDTPD) bonds in each molecule. The ratios of homocoupling bonds are in parentheses. ${ }^{e}$ The average number of end groups in each molecule.

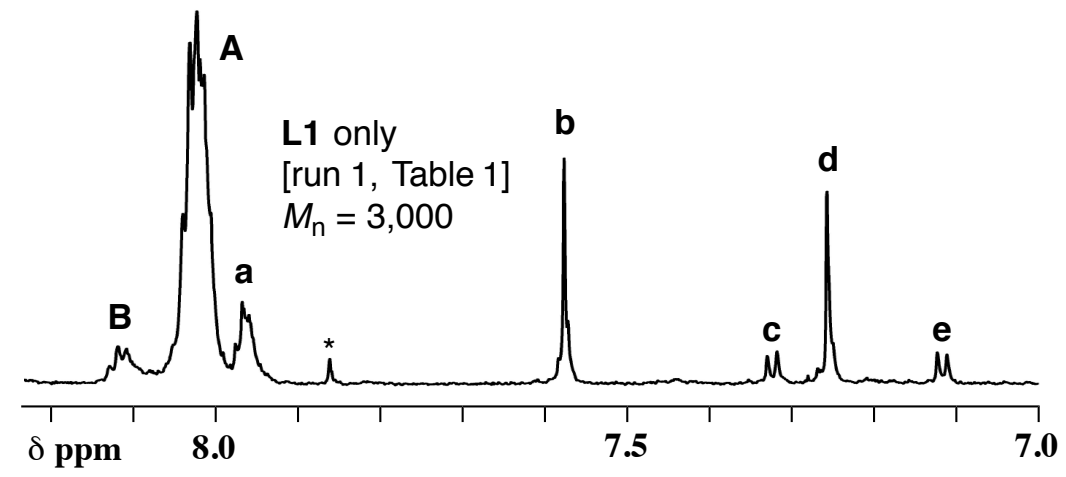

Figure S1. ${ }^{1} \mathrm{H}$ NMR spectrum of the DArP product of run 1 in Table $1\left(\mathrm{C}_{2} \mathrm{D}_{2} \mathrm{Cl}_{4}, 130{ }^{\circ} \mathrm{C}, 400 \mathrm{MHz}\right)$. *Free TPD- $\mathrm{H}_{2}$. 


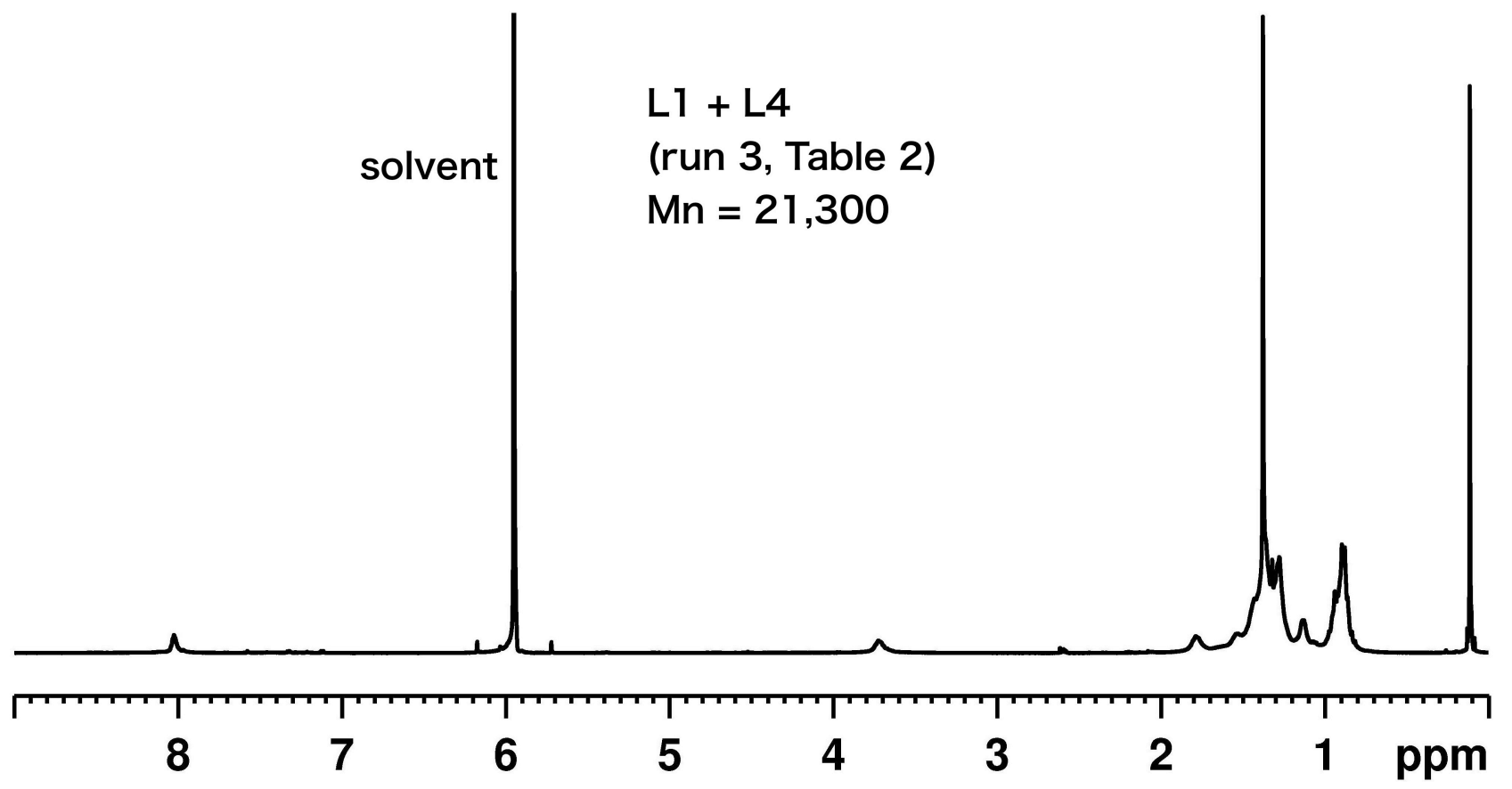

Figure S2. ${ }^{1} \mathrm{H}$ NMR spectrum of the DArP product of run 3 in Table $2\left(\mathrm{C}_{2} \mathrm{D}_{2} \mathrm{Cl}_{4}, 130{ }^{\circ} \mathrm{C}, 400 \mathrm{MHz}\right)$.

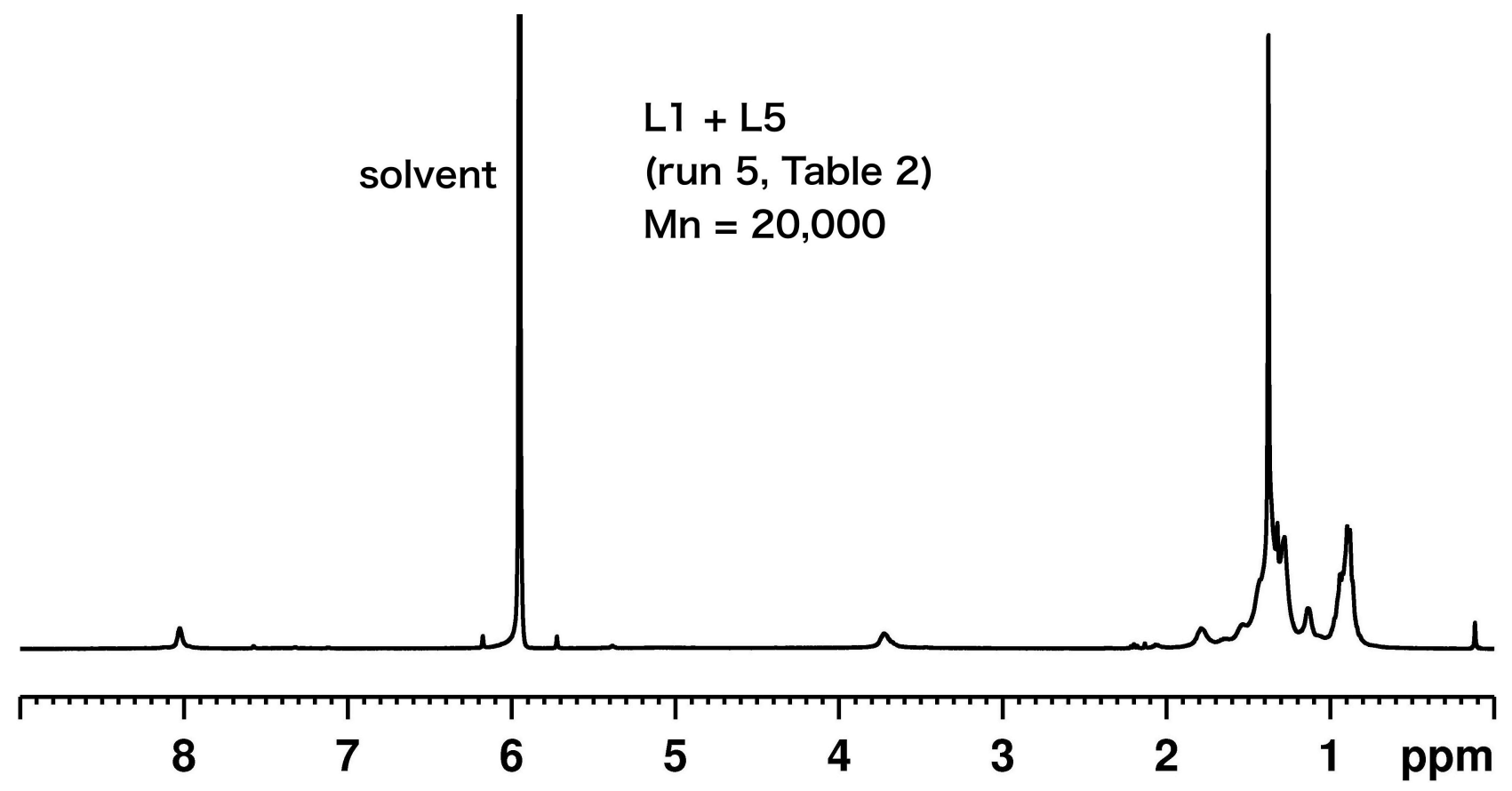

Figure S3. ${ }^{1} \mathrm{H}$ NMR spectrum of the DArP product of run 5 in Table $2\left(\mathrm{C}_{2} \mathrm{D}_{2} \mathrm{Cl}_{4}, 130{ }^{\circ} \mathrm{C}, 400 \mathrm{MHz}\right)$. 